\title{
A lung granuloma with partial anomalous pulmonary venous connection and bronchial atresia
}

\author{
K. Sumida, T. Kohno, MD, M. Mun, MD, A. Hamamoto, MD, T. Yoshiya, MD, and T. Takahashi, MD, Tokyo, Japan
}

$\mathrm{P}$ artial anomalous pulmonary venous connection (PAPVC) is an uncommon congenital anomaly that is physiologically unimportant in most asymptomatic patients. However, PAPVC can be problematic after major lung resection because of right ventricular failure owing to right ventricular volume overload caused by the left-to-right physiologic shunt. ${ }^{1}$ Bronchial atresia is also a rare congenital anomaly that may be found incidentally on chest radiographs in asymptomatic older children or adults. Patients with bronchial atresia may have fever, cough, or shortness of breath owing to postobstructive infections that may be recurrent. ${ }^{2}$ Herein we describe the case of a patient with lung granuloma suspected to be a pulmonary sequestration who was incidentally found to have PAPVC and bronchial atresia.

\section{Clinical Summary}

A 44-year-old woman visited her general practitioner because of a dry cough persisting for 2 months. Chest computed tomography (CT) showed a pulmonary tumor $7 \mathrm{~cm}$ in diameter in the left lower lobe. A bronchoscopic examination showed no abnormalities except for the lack of an entrance to the superior branch of the left lower lobe (B6). She was subsequently referred to our hospital for further examination. Contrast-enhanced chest CT showed a radiolucent area in the superior segment of the left lower lobe and a well-defined pulmonary tumor $(7 \mathrm{~cm}$ in diameter) in the lower segment of the same lobe (Figure 1, $A$ and $B$ ). Three-dimensional contrast-enhanced chest $\mathrm{CT}$ revealed an anomalous pulmonary vein originating from the hilum of the left lower lobe and draining into the azygos vein (Figure 1, C). The abnormality was diagnosed as a PAPVC of the left lower pulmonary vein to the azygos vein, accompanied by a pulmonary tumor and bronchial atresia of the superior branch of the left lower lobe.

Lobectomy of the left lower lobe was performed with videoassisted thoracic surgery for diagnostic and therapeutic purposes because a malignant or infectious tumor could not be ruled out. All procedures were performed with three access ports with doublelung ventilation and without minithoracotomy. The anomalous pulmonary vein was found on the left hilum, passing behind the

From the Department of Thoracic Surgery, Toranomon Hospital, Tokyo, Japan.

Received for publication April 23, 2007; revisions received July 22, 2007; accepted for publication Aug 14, 2007

Address for reprints: Keiichi Sumida, Department of Thoracic Surgery, Respiratory Center, Toranomon Hospital, 2-2-2, Toranomon, Minato-ku,

Tokyo, Japan (E-mail: k_sumida0504@yahoo.co.jp).

J Thorac Cardiovasc Surg 2008;135:449-50

$0022-5223 / \$ 34.00$

Copyright (C) 2008 by The American Association for Thoracic Surgery doi:10.1016/j.jtcvs.2007.08.050 descending aorta, toward the right side. The bronchial lumen was observed bronchoscopically, but we were unable to locate the entrance of the superior branch into the left lower lobe. Resection of the left lower lobe was successful with an uneventful postoperative course, and the patient was discharged on the fourth postoperative day.

Macroscopically, an emphysematous lesion was noted on the superior segment of the left lower lobe and a homogeneous, white solid tumor was localized in the medial side of the basal segment. The tumor had a bronchial structure, but the proximal part of the bronchus had blind ends and the connecting branch could not be detected. Histologic examination revealed fibrosis adjacent to the tumor and surrounding small muscular arteries, allowing possible drainage into the tumor from the descending aorta through the pulmonary ligament. An organized alveolar structure was retained in the tumor, epithelioid granuloma with infiltration of neutrophils and polynuclear giant cells was observed, and acid-fast bacteria in the granuloma were detected in Ziehl-Neelsen staining (Figure 2). The parenchyma was emphysematous in the superior segment of the left lower lobe, and dilated, mucus-filled bronchi were evident.

Results from macroscopic and histologic studies yielded a definitive diagnosis of granuloma with PAPVC and bronchial atresia.

\section{Discussion}

PAPVC is an uncommon congenital anomaly that is found in $0.5 \%$ to $0.7 \%$ of postmortem examinations. Anomalous drainage from the left side is even less common, occurring 10 times less frequently than from the right. ${ }^{3}$ There have been few reports of PAPVC in combination with bronchial atresia, and most asymptomatic PAPVCs are detected as incidental radiologic findings. However, adult patients with untreated PAPVC may eventually have severe pulmonary artery hypertension and markedly increased pulmonary vascular obstructive disease. ${ }^{4}$

Bronchial atresia is also a rare congenital anomaly. It was first reported in 1953, and approximately $50 \%$ of cases are found incidentally on chest radiographs in asymptomatic older children or adults. Infection is unusual because there is no communication with the normal tracheobronchial tree. The other $50 \%$ of patients have pulmonary symptoms such as fever, cough, or shortness of breath owing to recurrent pulmonary infection or hyperinflation of associated lung parenchyma. Diagnosis can often be based on radiographic features ${ }^{5}$ : chest radiograph and CT show increased tubular branching density reflecting mucus impaction or mucocele with surrounding focal hyperinflation. ${ }^{2}$

The lung granuloma contained bronchi with proximal blind ends and small muscular arteries around it. It was unclear whether the muscular arteries developed secondarily to inflammation of granuloma, suggesting the original presence of another bronchial atresia.

In our patient, dry cough was the only preoperative symptom that might have resulted from either bronchial atresia or PAPVC. If the 

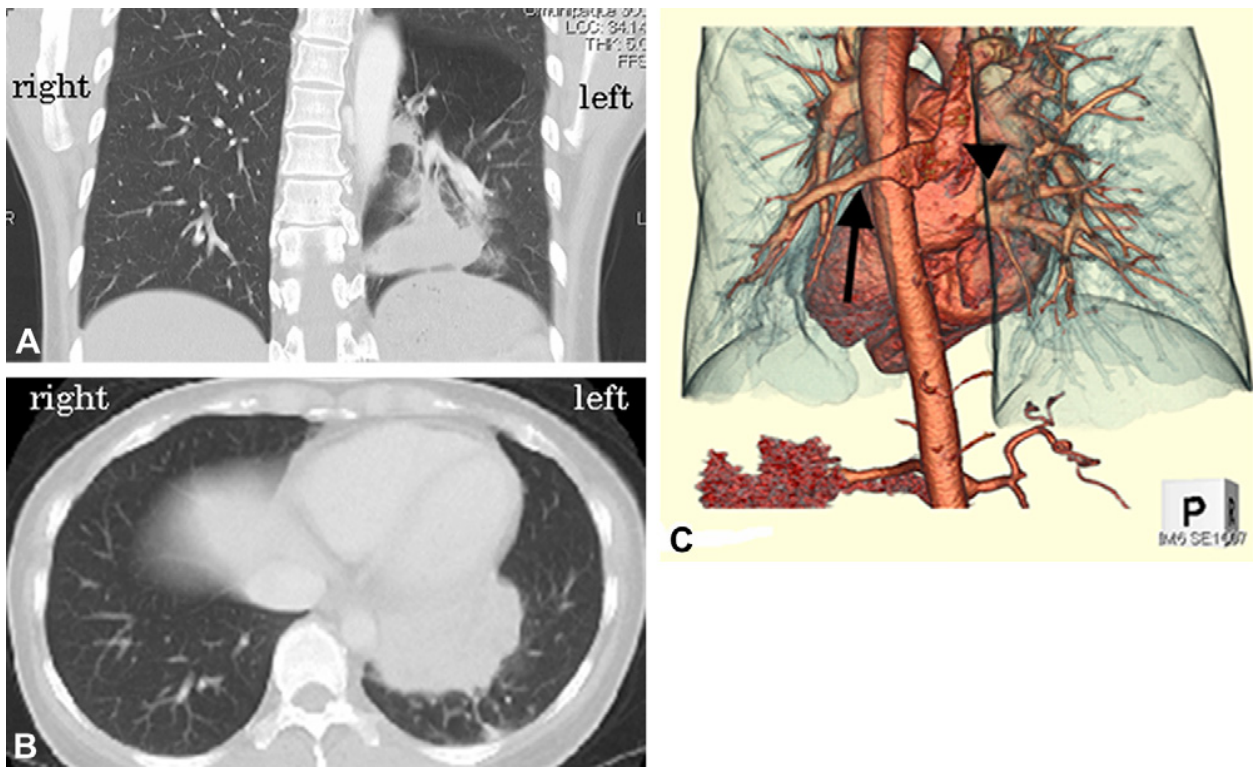

Figure 1. A, A chest CT scan of the coronal view showing a radiolucent area in the superior segment of the left lower lobe and a well-defined pulmonary granuloma in the lower segment of the same lobe. B, A chest CT scan of the axial view showing a pulmonary granuloma in the lower segment. C, A 3-dimensional CT scan from the posterior view showing an anomalous pulmonary vein (arrow) originating from the hilum of left lower lobe and draining into the azygos vein (arrowhead).

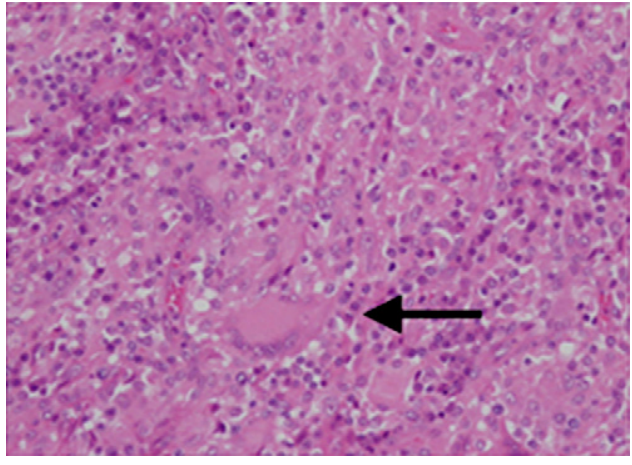

Figure 2. Epithelioid granuloma with infiltration of neutrophils and polynuclear giant cells was seen (arrow). Acid-fast bacteria were identified in Ziehl-Neelsen staining of the granuloma. However, a polymerase chain reaction study of bacterial cultures gave negative results for Mycobacterium tuberculosis complex, Mycobacterium avium, and Mycobacterium intracellulare.
PAPVC had not originated from the left lower lobe with the lung tumor, an additional operation to correct the anomalous vein might have been needed to prevent right ventricular failure. Fortunately, the PAPVC and bronchial atresia coexisted with the lung granuloma, so that left lower lobectomy was sufficient to cure all the anomalies.

\section{References}

1. Takei H, Suzuki K, Asamura H, Kondo H, Tsuchiya R. Successful pulmonary resection of lung cancer in a patient with partial anomalous pulmonary venous connection: report of a case. Surg Today. 2002;32: 899-901.

2. Nordstrom CR, Kane GC, Wechsler RJ, Salazar AM, Cohn HE, Farber JL. Bronchial atresia with relapsing pulmonary infection in a middle-aged man. Respir Care. 2001;46:601-3.

3. Neirotti R, Gonzalez-Lavin L, Rose DN. Anomalous pulmonary venous connection of the left lung associated with valvular heart disease: report of two cases. Br Heart J. 1972;34:969-73.

4. Bobb JD, McGlynn TJ, Pierce WS, Kirkman PM. Isolated partial anomalous venous connection: a congenital defect with late and serious complications. Ann Thorac Surg. 1981;31:540-3.

5. Murat A, Ozdemir H, Yildirim H, Kursad Poyraz A, Artas H. Bronchial atresia of the right lower lobe. Acta Radiol. 2005;46:480-3. 